\title{
Striking a balance between treatment benefits and toxicity in older women with
} early-stage breast cancer

\section{“Early-stage breast cancers that are newly diagnosed in elderly women tend to have favorable pathologic features compared with younger women."}

This issue of Aging Health is dedicated to the theme of oncology. Worldwide, breast cancer is the most common malignancy in women, and generally is a disease of older women. Despite improvements in the treatment of early-stage breast cancer, there remains a disproportionately high death rate from breast cancer in the elderly population [1]. The cause for this discrepancy is likely to be multifactorial. For example, it has been shown that older women present with more advanced disease at diagnosis. Compared with younger women, those over the age of 70 years are more likely to present with axillary nodal involvement at their initial diagnosis of breast cancer $[2,3]$. Competing comorbidities contribute to the individual patient's outcome and may compromise the ability to administer full doses of adjuvant therapy. Using case examples, survey data suggest that medical oncologists are cautious in recommending adjuvant therapy in women of advancing age or poorer health status [4]. The progress that is being made in the treatment of older women with breast cancer serves as an example of how geriatricians are influencing the field of oncology. For more than a decade, a partnership between the American Society of Clinical Oncology (ASCO; VA, USA) and the Hartford Foundation (NY, USA) has fostered a true 'geriatric oncology movement' that is dedicated to the improvement of cancer care in this population. Geriatric symposia have been sponsored and the national hematology/oncology meetings dedicate educational and scientific sessions to geriatric themes. The Hartford Foundation is working together with the ASCO and the Association of Subspecialty Professors in Medicine (ASP; VA, USA) sponsored joint geriatric and medical oncology training. A core group of geriatric oncologists are working to initiate and direct trials that address clinical problems unique to this population, consolidating patient resources and providing education to nongeriatric physicians so that they can better care for elderly patients with cancer. The impact and importance of geriatric oncology is exemplified in the management of early-stage breast cancer.

The interdisciplinary treatment of early-stage breast cancer with surgery, radiation therapy, chemotherapy and/or endocrine therapy has improved the cure rate of women with early-stage breast cancer. Younger women who have newly diagnosed breast cancer focus on the prolongation of life and will opt to receive chemotherapy even if the potential benefit to them is only $1 \%$. For older women, the threshold for receipt of adjuvant treatment may differ, as the potential impact of therapy on quality of survival may play as important a role as length of survival. Recommendations for medical interventions in this population strike a balance between the benefits and toxicity of treatment.

"There will come a day when the genetic profiles of the patient and their tumor will help to define the course of cancer treatment."

Personalized therapy has become a buzz word in medicine. In oncology, the term is generally used to describe cancer treatments that are tailored to the patient based on their unique malignant disease process. In reality, we have had individualized cancer therapy for women with breast cancer for many years. Systemic treatment recommendations are based on the primary tumor's hormone receptor status (estrogen and progesterone receptors), menopausal status and HER2 status. The geriatric philosophy of patient care adds an additional component to individualized care, through understanding the physiologic age of the patient and how this might impact the risks and benefits of treatment

Early-stage breast cancers that are newly diagnosed in elderly women tend to have favorable pathologic features compared with younger
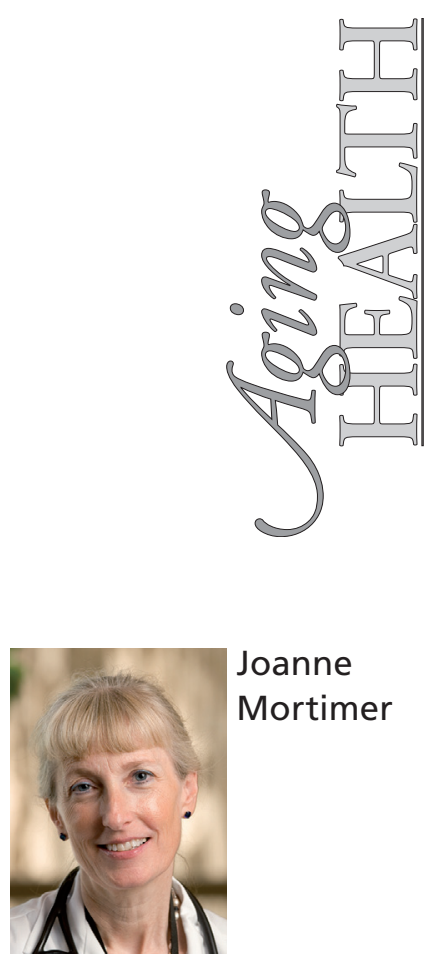

Author for correspondence: City of Hope, 1500 East Duarte Road, Duarte, CA 91010, USA Jmortimer@coh.org

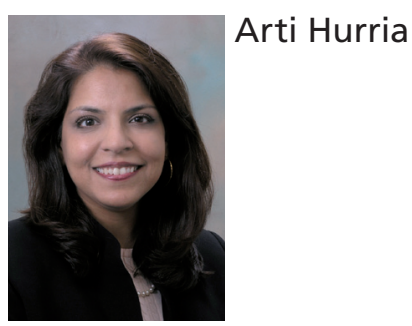

City of Hope, 1500 East Duarte Road, Duarte, CA 91010, USA

\section{Keywords}

- coming of age

- geriatric oncology 
women. The tumors are more likely to be well differentiated, hormone dependent and HER 2 negative [5]. Therefore, endocrine therapy is an important component of the treatment. One of the early trials that addressed a personalized approach to elderly women with early-stage breast cancer asked the question, do all women with localized earlystage hormone-dependent breast cancer require radiation therapy after lumpectomy? A large cooperative group trial enrolled women aged 70 years and older, who had undergone a lumpectomy for a hormone receptor-positive tumor that was smaller than $2 \mathrm{~cm}$, and who had clinically negative axillary lymph nodes. Patients were randomized to receive tamoxifen alone or conventional whole breast irradiation and tamoxifen. A small increase in local breast cancer recurrences was observed in those women assigned to receive tamoxifen alone. However, the increase in local disease recurrence did not impact overall survival. The initial findings were reported with 5 years median follow-up. With 12 years of median follow-up, the data still indicate that the addition of radiation therapy to tamoxifen does not impact overall survival in this subset of patients [6,7].

"Systemic treatment
recommendations are based on the
primary tumor's hormone receptor
status (estrogen and progesterone
receptors), menopausal status and
HER2 status."

Another pivotal trial addressed the question, do older women require the same aggressive chemotherapy as younger women? Once again, the cooperative groups collaborated to enroll women $\geq 65$ years with stage I-III invasive ductal cancer. Participants were randomized to receive either the oral agent, capecitabine, for 6 months or conventional chemotherapy (either cyclophosphamide, methotrexate and 5-fluorouracil or cyclophosphamide and doxorubicin). A prespecified data analysis was planned with enrollment of the 600th patient and a statistically significant improvement in both disease-free and overall survival was observed in the women assigned conventional therapy [8]. This confirmed that aggressive cancers require more aggressive and potentially more toxic chemotherapy, even among older adults with breast cancer. But how do we know which patients are able to tolerate aggressive chemotherapy regimens?

The Comprehensive Geriatric Assessment is the hallmark of the geriatrician. In geriatric oncology, studies are underway to test clinical tools that will predict which patients experience unacceptable toxicity with therapeutic intervention $[9,10]$. Once these tools are validated and applied, we will have additional methods for individualizing cancer care in the elderly patient. These tools are being tested and validated in clinical settings using electronic data capture systems. Such technology will provide even the nongeriatric physician with an efficient and portable way of assessing physiologic age. Such information will help to identify which patients can be safely treated with aggressive chemotherapy and which patients will experience unacceptable side effects. There will come a day when the genetic profiles of the patient and their tumor will help to define the course of cancer treatment. Until then, incorporation of the geriatric philosophy into the care of cancer patients has greatly improved our ability to provide personalized care.

\section{Financial \& competing interests disclosure}

The authors have no relevant affiliations or financial involvement with any organization or entity with a financial interest in or financial conflict with the subject matter or materials discussed in the manuscript. This includes employment, consultancies, honoraria, stock ownership or options, expert testimony, grants or patents received or pending, or royalties.

No writing assistance was utilized in the production of this manuscript.

\section{Bibliography}

1. Wildiers H, Kunkler I, Biganzoli L et al.: Management of breast cancer in elderly individuals: recommendations of the International Society of Geriatric Oncology. Lancet Oncol. 8(12), 1101-1115 (2007).

2. Wildiers H, Van Calster B, van de PollFranse LV et al.: Relationship between age and axillary lymph node involvement in women with breast cancer. J. Clin. Oncol. 27(18), 2931-2937 (2009).
3. Schonberg MA, Marcantonio ER, Li D, Silliman RA, Ngo L, McCarthy EP: Breast cancer among the oldest old: tumor characteristics, treatment choices, and survival. J. Clin. Oncol. 28(12), 2038-2045 (2010).

4. Hurria A, Wong FL, Villaluna D et al.: Role of age and health in treatment recommendations for older adults with breast cancer: the perspective of oncologists and primary care providers. J. Clin. Oncol. 26(33), 5386-5392 (2008).
5. Diab SG, Elledge RM, Clark GM: Tumor characteristics and clinical outcome of elderly women with breast cancer. J. Natl Cancer Inst. 92(7), 550-556 (2000).

6. Hughes KS, Schnaper LA, Berry D et al.: Lumpectomy plus tamoxifen with or without irradiation in women 70 years of age or older with early breast cancer. $N$. Engl. J. Med. 351(10), 971-977 (2004).

7. Hughes KS, Schnaper LA, Cirrincione C et al.: Lumpectomy plus tamoxifen with or without irradiation in women age 70 or 
older with early breast cancer. J. Clin. Oncol. 28(Suppl. 15), 507 (2010).

8. Muss HB, Berry DA, Cirrincione CT et al.: Adjuvant chemotherapy in older women with early-stage breast cancer. $N$. Engl. J. Med. 360(20), 2055-2065 (2009).
9. Hurria A, Togawa K, Mohile SG et al.: Predicting chemotherapy toxicity in older adults with cancer: a prospective 500 patient multicenter study. J. Clin. Oncol. 28(Suppl. 15) (2010) (Absract 9001).
10. Extermann M, Boler I, Reich R et al:: The Chemotherapy Risk Assessment Scale for High-Age Patients (CRASH) score: design and validation. J. Clin. Oncol. 28(Suppl. 15) (2010) (Abtract 9000). 